\title{
EXPRESSION OF HUMAN UBIQUITOUS AQUAPORINS IN CHORIAL VILLUS SAMPLES
}

J. Escobar ${ }^{1}$, A. Arduini ${ }^{2}$, M. Gormaz ${ }^{3}$, K. Gosens ${ }^{4}$, A. Martinez ${ }^{5}$, A. Perales ${ }^{5}$, R. Escrig ${ }^{3}$, E. Tornos ${ }^{5}$, M. Vento $^{3,6}$

${ }^{1}$ Health Research Institute Hospital La Fe, Hospital Universitario y Politécnico La FE, ${ }^{2}$ Department of Physiology, University of Valencia, ${ }^{3}$ Division of Neonatology, University and Polytechnic Hospital La Fe, Valencia, Spain, ${ }^{4}$ Obstetrics, Radboud University Nijmegen Medical Center, Nijmegen, Netherlands Antilles, ${ }^{5}$ Obstetrics, ${ }^{6}$ Health Research Institute (I.I.S. La Fe), University and Polytechnic Hospital La Fe, Valencia, Spain

Background/objectives: Aquaporins (AQPs) are a family of proteins (AQP0-12) ubiquitously expressed acting as cell membrane water channels. AQP 1/3/8/9 expression has been found in human placenta and fetal membranes; however, AQP4 is the only identified in first trimester fetal tissue samples. We aimed to determine AQP mRNA expression in first trimester of pregnancy and compare it to the expression in placenta at delivery.

Material and methods: 26 Chorionic villus (CV) samples and 5 placental samples were collected and analyzed by real time-PCR using Taqman assay (Applied Biosystems ${ }^{\circledR}$ ) for human AQP1, 2, 3, 4, 5, 6, 7, 8, 9,11 and $18 \mathrm{~S}$.

Results: CV expressed high mRNA levels of AQP1, 3, 9 and 11. Expression of AQP11 is described for the first time in literature. AQP9 and 11 mRNA expression was significantly reduced in term placenta as compared to $\mathrm{CV}$. Conversely, CV expressed low mRNA for AQP4, 5 and 8 while significantly higher expression was detected in term placenta. AQP2/6/7 were not expressed in CV or term placenta.

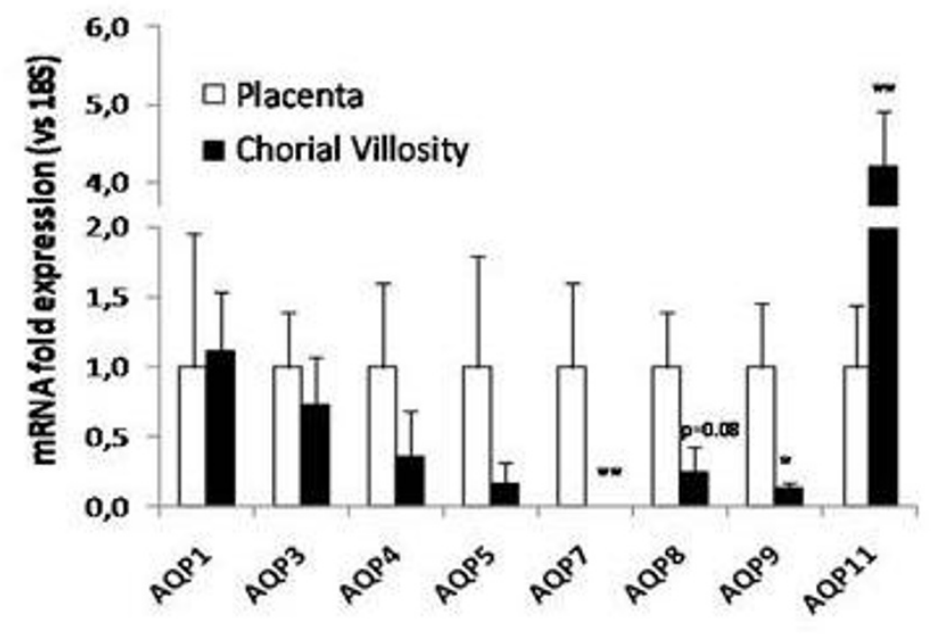

[Aquaporins' mRNA expression in human pregnancy]

Conclusions: There are significant differences between expression of AQPs in the first and third trimesters of pregnancy. Expression of AQP11 in the first trimester has been described for the first time in literature. Data suggest that AQP 9 and 11 could play an important role for fetal development. 